\title{
The cosmic ray ionization rate in the central parsec of the Galaxy
}

\author{
Miwa Goto \\ Universitäts-Sternwarte München, Scheinerstr. 1, D-81679 Munich, Germany \\ email: mgoto@usm.lmu.de
}

\begin{abstract}
Cosmic rays represent a unique crossing point of high-energy astrophysics and astrochemistry. The cosmic ray ionization rate of molecular hydrogen $\left(\zeta_{2}\right)$ measured by $\mathrm{H}_{3}^{+}$spectroscopy in the central parsec of the Galaxy is 2 orders of magnitude higher than that in the dense clouds outside the Galactic center. However, it is still too short, by the factor of 10,000, to agree with an extremely high $\zeta_{2}$ that accommodates the new $\gamma$-ray observations of Sgr A* and its environment.
\end{abstract}

Keywords. Galaxy: center - cosmic rays - astrochemistry - ISM: clouds - ISM: lines and bands - ISM: molecules — stars: individual (GCIRS 1W, GCIRS 3)

\section{Cosmic rays}

Cosmic rays are the high energy particles $(E>\mathrm{MeV})$, mostly consisting of protons, that precipitate on the Earth's atmosphere every second. The cosmic rays are a subject of interest not only in high-energy astrophysics, but also in low-energy astrochemistry, because they are the source of ionization in dark clouds. In the cold interstellar medium, neutral-neutral reactions are prohibitively slow, because of their reaction barriers. Ionneutral reactions that proceed with high Langevin rates are the main propellant of the chemistry in the ISM. Deep inside a molecular cloud, where ionizing UV photons from the interstellar radiation field are attenuated, the cosmic rays are the sole source of ionization that trigger the ion-neutral reactions. The production of interstellar molecules is sometimes strongly dependent on the ionization of the seed molecules by cosmic rays. The abundance of such molecules serves as an excellent measure of the cosmic ray flux in the medium in question.

$\mathrm{H}_{3}^{+}$is the best chemical probe of the cosmic rays, because the number of the reactions involved is virtually one (Oka 2006). The formation of $\mathrm{H}_{3}^{+}$starts with the ionization of molecular hydrogen by a cosmic ray. $\mathrm{H}_{2}^{+}$is unstable, and quickly reacts with another molecular hydrogen to form $\mathrm{H}_{3}^{+}$. This reaction is faster than other competing processes; every $\mathrm{H}_{2}^{+}$formed eventually turns to $\mathrm{H}_{3}^{+}$under normal dense cloud conditions. Therefore the abundance of $\mathrm{H}_{3}^{+}$is a direct measure of the efficiency of the first path, the ionization rate of $\mathrm{H}_{2}$ by a cosmic ray.

High angular resolution $\gamma$-ray telescopes recently online provide direct estimates of the high-energy cosmic ray flux in the interstellar medium. The underlying assumption is that $\mathrm{TeV} \gamma$-rays are produced by neutral pion decays following the collisions of accelerated protons with the ambient cold nuclei. In this contribution we will compare the cosmic ray ionization rate of molecular hydrogen $\zeta_{2}$ in the central parsecs of our Galaxy measured in two ways: one by the means of astrochemistry using $\mathrm{H}_{3}^{+}$spectroscopy, and the other by proton injection model to reproduce the observed $\gamma$-ray spectrum discovered near Sgr A*. 


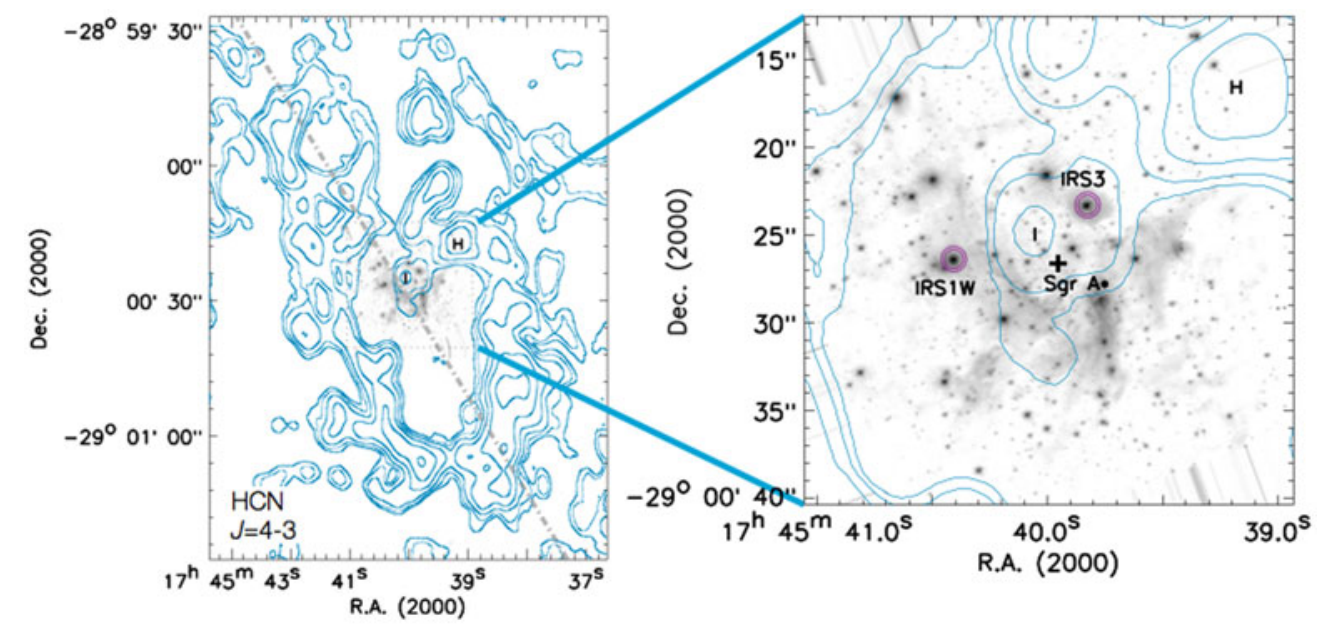

Figure 1. HCN $J=(4-3)$ map of the circumnuclear ring (left, blue contours; Montero-Castaño et al. 2009), and a blow-up view of the central part (right) overlaid with the near-infrared ( $K$-band) image of the Central cluster. The height of the field of the right panel is 5 pc. The clumps identified by Montero-Castaño et al. (2009) are labeled with I and H. [A COLOR VERSion is AVAILABLE ONLINE.]

\section{Laboratory}

The Galactic center is very energetic and contains vast reservoirs of molecular gas at the same time. One tenth of the molecular mass of whole Milky Way is supposedly concentrated in the central molecular zone (CMZ) in $200 \mathrm{pc}$ of the Galactic center (Morris \& Serabyn 1996). On the other hand, supernova outbursts happen 2000 times more frequently than outside the Galactic center (Crocker et al. 2011). The Galactic center harbors plenty of bright infrared sources that enable high-precision infrared absorption spectroscopy. The Galactic center is a unique laboratory to study how a high energy $\mathrm{x}$-ray and $\gamma$-ray sources have a direct impact on the chemistry.

Figure 1 shows a near-infrared image of the Central cluster of our Galaxy overlaid with the circumnuclear ring (CNR) mapped by $\mathrm{HCN} J=(4-3)$ (Montero-Castaño et al. 2009). Note that the line of sight to the bright infrared source GCIRS 3 overlaps with a clump in the CNR, while the line of sight to another member of the Central cluster GCIRS $1 \mathrm{~W}$ is clear. The absorption toward the Galactic center is the sum total of the absorption along the line of sight over $8 \mathrm{kpc}$, including diffuse molecular clouds in the $\mathrm{CMZ}$, and the dense clouds in the foreground spiral arms. In order to pick up exclusively $\mathrm{H}_{3}^{+}$absorption in the dense clump in the $\mathrm{CNR}$, the $\mathrm{H}_{3}^{+}$spectrum of GCIRS $1 \mathrm{~W}$ was subtracted from that of GCIRS 3. The projected distance of the two sources is $0.3 \mathrm{pc}$. The foreground absorption that takes place in the common path should be canceled out.

The $\mathrm{H}_{3}^{+}$spectra from the rotational levels $J=1,2,3$ obtained by CRIRES spectrograph at the Very Large Telescope are shown in Figure 2. GCIRS 3 shows excess absorption at the positive velocity. The line profile of HCN $J=(4-3)$ extracted at the position of GCIRS 3 matches well to $\mathrm{H}_{3}^{+} R(2,2)^{l}$. The agreement lends convincing support to the idea that the excess absorption indeed arises in the clump local to the CNR. The column density of $\mathrm{H}_{3}^{+}$required to reproduce the excess absorption is $N\left(\mathrm{H}_{3}^{+}\right)=6 \times 10^{14} \mathrm{~cm}^{-2}$, which is $\zeta_{2}>1.6 \times 10^{-15} \mathrm{~s}^{-1}$, if the pathlength of the cloud is smaller than the projected distance between GCIRS 3 and GCIRS 1W (Goto et al. 2013a; Goto et al. 2013b). This cosmic ray ionization rate measured is the closest measured rate in position to Sgr $\mathrm{A}^{*}$ by 


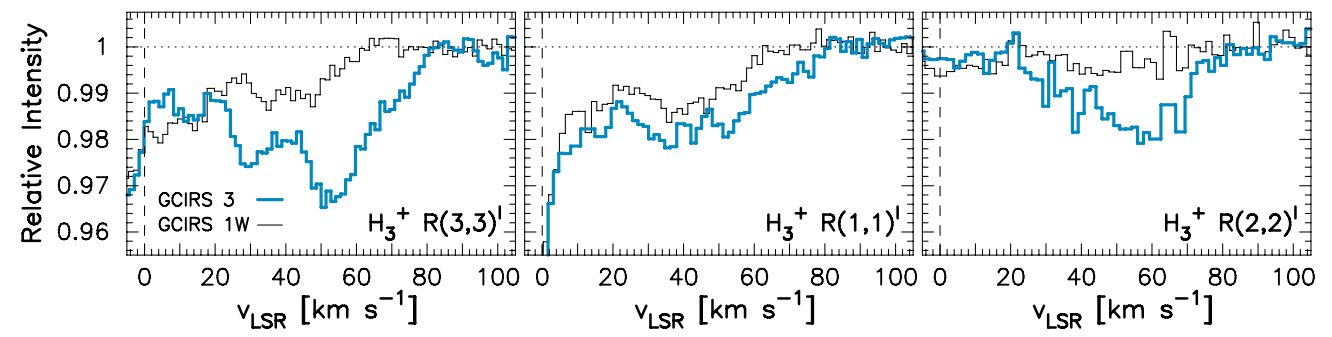

Figure 2. Infrared $\mathrm{H}_{3}^{+}$absorption spectra of GCIRS 3 and GCIRS $1 \mathrm{~W}$.

[A COLOR VERSION IS AVAILABLE ONLINE.]

the astrochemistry, and is two orders of magnitude higher than that of the dense clouds outside the Galactic center (Figure 3).

\section{3. $\gamma$-ray observations}

A TeV $\gamma$-ray source HESS $1745-290$ was discovered by the HESS collaboration in the Galactic center (Aharonian et al. 2004), and subsequently localized to a few arcminutes of Sgr A*. The proton flux required to reproduce the $\gamma$-ray spectra observed by the HESS and the Fermi/LAT is $7 \times 10^{4}$ proton $\mathrm{cm}^{-2} \mathrm{sr}^{-1} \mathrm{~s}^{-1}(\mathrm{GeV} / \text { nucleon })^{-1}$ at the energy $1 \mathrm{GeV}$ in 10 pc of Sgr A* (Chernyakova et al. 2011). This is 100,000 times more than the cosmic ray flux in the solar neighborhood at the same energy. If we take the cosmic ray spectrum proposed by Indriolo et al. (2009) at the extrapolation of the flux to MeV regime, the cosmic ray ionization rate of $\mathrm{H}_{2}$ comes up to $1.1 \times 10^{-11} \mathrm{~s}^{-1}$, which is 10,000 times larger than that we found by $\mathrm{H}_{3}^{+}$spectroscopy at the same place.

\section{Possible explanations}

There are at least three issues that might be treated incorrectly in the calculations above. First, we extrapolated the cosmic ray spectrum over 3 orders of magnitudes from $\mathrm{GeV}$ to $\mathrm{MeV}$ to calculate $\zeta_{2}$ from $\gamma$-ray observation. TeV- $\gamma$ ray spectroscopy provides a constraint on the cosmic ray flux down to $\sim 1 \mathrm{GeV}$, while the cosmic rays that affect $\zeta_{2}$ most are of $\lesssim \mathrm{MeV}$, because the ionization cross section of $\mathrm{H}_{2}$ peaks at $\sim 100 \mathrm{keV}$. We used the cosmic ray spectrum of Indriolo et al. (2009), which is consistent with the latest $\mathrm{H}_{3}^{+}$spectroscopy in the Galactic diffuse clouds; but other choices of spectra with strong turnovers in low-energy regime (e.g. Webber 1998) reduce $\zeta_{2}$ significantly. In-situ measurements of the cosmic ray spectrum by Voyagers outside the heliosphere is awaited (Stone et al. 2013; Webber et al. 2013).

Second, the dissociative recombination of $\mathrm{H}_{3}^{+}$with the electrons may not be negligible even in a dense cloud, if the cosmic ray ionization rate is significantly larger than $10^{-15} \mathrm{~s}^{-1}$. The crucial assumption that leads $\mathrm{H}_{3}^{+}$abundance to be proportional to $\zeta_{2}$ is that the destruction of $\mathrm{H}_{3}^{+}$happens primarily through the chemical reaction with $\mathrm{CO}$ in dense clouds. This is a reasonable assumption in normal dense clouds where the electron density is much smaller than the $\mathrm{CO}$ abundance. If, however, the dissociative recombination is not negligible, the abundance of $\mathrm{H}_{3}^{+}$becomes smaller, and $\zeta_{2}$ calculated with the incorrect premise is subject to a substantial underestimation.

The third possibility that I learned during the symposium is the possibility of a source misidentification with the pulsar wind nebula G359.95-0.04 (Wang et al. 2006). If a part of $\gamma$-ray emission observed by HESS is attributed to the inverse Compton scattering by 


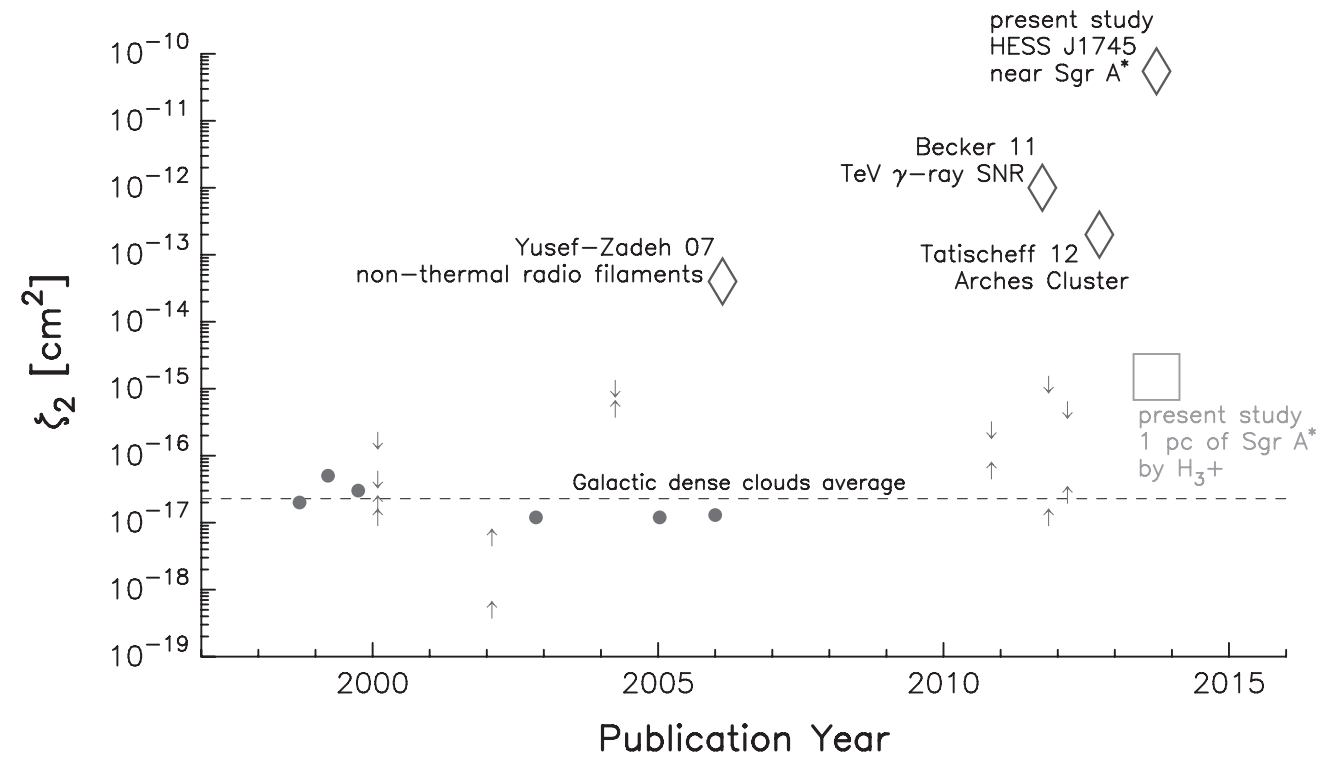

Figure 3. The history of the measurements of the cosmic-ray ionization rate by astrochemical probes in dense clouds (filled circle; Williams et al. 1998; McCall et al. 1998; Bergin et al. 1999; Padoan et al. 2004; Lee et al. 2004; Wakelam et al. 2005), and in the Galactic center from the present study. The open diamonds are the recent predictions of $\zeta_{2}$ from high-energy observations (Yusef-Zadeh et al. 1007; Becker et al. 2011; Tatischeff et al. 2012).

electrons accelerated in the pulsar wind (Hinton \& Aharonian 2007), the proton flux calculated by Chernyakova et al. (2011) may be reduced significantly.

An extreme $\zeta_{2}\left(>10^{-14} \mathrm{~s}^{-1}\right)$ predicted by high-energy observations either in x-rays or $\gamma$-rays is not an exception any more (Figure 3 ). Such a high $\zeta_{2}$ has a huge impact on the chemistry. However, there is a gap between the observations and the predictions as is shown in the present study. Further $\mathrm{H}_{3}^{+}$observations with better spatial sampling in the CNR should be in order to find an experimental solution to the problem.

\section{References}

Aharonian, F., Akhperjanian, A. G., Aye, K.-M., et al. 2004, A\&A 425, L13

Becker, J. K., Black, J. H., Safarzadeh, M., \& Schuppan, F. 2011, ApJ Lett. 739, L43.

Bergin, E. A., Plume, R., Williams, J. P., \& Myers, P. C. 1999, ApJ 512, 724

Chernyakova, M., Malyshev, D., Aharonian, F. A., Crocker, R. M., \& Jones, D. I. 2011, ApJ 726,60 .

Crocker, R. M., Jones, D. I., Aharonian, F., Law, C. J., Melia, F., Oka, T., \& Ott, J. 2011, MNRAS 413, 763

Goto, M., Indriolo, N., Geballe, T. R., \& Usuda, T. 2013, JPC A 117, 9919

Goto, M., Geballe, T. R., Indriolo, N., Yusef-Zadeh, F., Usuda, T., Henning, Th. \& Oka, T. 2013, ApJ, submitted

Hinton, J. A. \& Aharonian, F. A. 2007, ApJ 657, 302

Indriolo, N., Fields, B. D., \& McCall1 , B. J. 2009, ApJ 694, 257

Lee, J.-E., Bergin, E. A., \& Evans, N. J. 2004, ApJ 617, 360

McCall, B. J., Geballe, T. R., Hinkle, K. H., \& Oka, T. 1999, ApJ 522, 338

Montero-Castaño, M., Herrnstein, R. M., \& Ho, P. T. P.. 2009, ApJ, 695, 1477

Morris, M. \& Serabyn, E. 1996, ARAA 34, 645

Oka, T. 2006, PNAS, 103, 12235

Padoan, P., Willacy, K., Langer, W., \& Juvela, M. 2004, ApJ 614, 203 
Stone, E. C., Cummings, A. C., McDonald, F. B., Heikkila, B. C., Lal, N., \& Webber, W. R. 2013, Science 341, 150

Tatischeff, V., Decourchelle, A., \& Maurin, G. 2012, A\&A 546, 88.

Wakelam, V., Selsis, F., Herbst, E., \& Caselli, P. 2005, A\&BA 444, 883

Wang, Q. D., Lu, F. J., \& Gotthelf, E. V. 2006, MNRAS 367, 937

Webber, W. R. 1998, ApJ 506, 329

Webber, W. R., Higbie, P. R., \& McDonald, F. B. 2013, arXiv:1308.1895

Williams, J. P., Bergin, E. A., Caselli, P., Myers, P. C., \& Plume, R. 1998, ApJ 503, 689

Yusef-Zadeh, F., Muno, M., Wardle, M., \& Lis, D. C. 2007, ApJ 656, 847 